\title{
Retinal degeneration: Multilevel protection of photoreceptor and ganglion cell viability and function with the novel PKG inhibitor CN238.
}

\section{Authors}

Arianna Tolone ${ }^{1+}$, Wadood $\mathrm{Haq}^{2+}$, Alexandra Fachinger ${ }^{3}$, Andreas Rentsch ${ }^{4}$, Friedrich W. Herberg $^{3}$, Frank Schwede ${ }^{4}$, François Paquet-Durand ${ }^{1 *}$

\section{Affiliations}

1 Cell Death Mechanism Group, Institute for Ophthalmic Research, Eberhard-Karls-Universität Tübingen, Germany.

2 Institute for Ophthalmic Research, Eberhard-Karls-Universität Tübingen, Germany.

3 Biochemistry Department, University of Kassel, Germany

4 Biolog Life Science Institute GmbH \& Co. KG, Bremen, Germany

+ these authors contributed equally

* To whom correspondence should be addressed.

\section{Abstract}

Hereditary retinal degeneration (RD) is often associated with excessive cGMP-signaling in photoreceptors. Previous research has shown that inhibition of cGMP-dependent protein kinase G (PKG) can slow down the loss of photoreceptors in different RD animal models. In this study, we identified a novel PKG inhibitor, the cGMP analogue CN238, with strong protective effects on photoreceptors in retinal degeneration $r d 1$ and $r d 10$ mutant mice. In long-term organotypic retinal explants, $\mathrm{CN} 238$ preserved $r d 1$ and $r d 10$ photoreceptor viability and function. Surprisingly, in explanted retinae CN238 also protected retinal ganglion cells from axotomy induced retrograde degeneration and preserved their functionality. Together, these results confirm the strong neuroprotective capacity of PKG inhibitors for both photoreceptors and retinal ganglion cells, thereby significantly broadening their potential applications for the treatment of retinal diseases and possibly neurodegenerative diseases in general.

\section{Keywords}

Apoptosis, neuroprotection, retinitis pigmentosa, cGMP, cGK1, functional vision preservation, micro-electrode array recording 


\section{Introduction}

Photoreceptor degeneration is a hallmark of retinal degenerative diseases (RD), a group of retinal dystrophies characterized by primary dysfunction and degeneration of photoreceptor cells, leading to visual loss and eventually to blindness (Berger, Kloeckener-Gruissem, and Neidhardt (2010). Because of the genetic heterogeneity of RD-type diseases, no effective therapies are currently available (Sahel, Marazova, and Audo 2014). While the mechanisms causing photoreceptor degeneration are far from being fully understood, high levels of cyclic guanosine monophosphate (cGMP) are known to trigger non-apoptotic photoreceptor cell death in many RD disease models (Arango-Gonzalez et al. 2014; Power et al. 2020). Two commonly used models for studying RD are $r d 1$ and $r d 10$ mice, which are characterized by a nonsense $(r d 1)$ and a missense $(r d 10)$ mutation in the gene encoding for the $\beta$-subunit of rod phosphodiesterase (PDE) 6 (Han et al. 2013). Lack of PDE6 activity leads to an accumulation of cGMP in photoreceptors (Farber and Lolley 1974; Paquet-Durand et al. 2009) and emerging $\mathrm{RD}$ neuroprotection strategies include targeting pathways downstream of cGMP (ArangoGonzalez et al. 2014; Power et al. 2020).

The prototypic cellular target of cGMP is protein kinase G (PKG), a serine/threonine kinase that exists as a homodimer of two subunits, each consisting of an $\mathrm{N}$-terminal dimerization domain, an auto-inhibitory sequence, two cGMP binding sites, and a C-terminal kinase domain. Three isoforms of PKG have been identified in mammals: PKG1 $\alpha$, PKG1 $\beta$ and PKG2. When $C G M P$ binds to PKG, it induces the release of the C-terminal catalytic domain from the auto-inhibitory sequence, activating PKG (Kim et al. 2016). cGMP-signaling activates PKG, which when overactivated is likely to play a key role in triggering cell death (Browning 2008; Power et al. 2020; Canals et al. 2003; Canzoniero et al. 2006; Fallahian et al. 2011; Leung et al. 2010). In the retina, exceedingly high cGMP levels, as well as strong PKG activation were causally linked to photoreceptor cell death (Paquet-Durand et al. 2009; Farber and Lolley 1974; Lolley et al. 1977), highlighting PKG as a target for the treatment of RD-type diseases.

In an effort to develop new drugs for the treatment of RD, a number of cGMP analogues designed to inhibit PKG have been synthetized (Butt, Eigenthaler, and Genieser 1994; Vighi, Trifunovic, et al. 2018). These compounds bear an Rp-configured phosphorothioate, which enables them to antagonize the activation of PKG by binding to the CGMP binding sites in the regulatory domain, without liberating the catalytic domain (Zhao et al. 1997). A previous study showed that the in vivo treatment with the cGMP analogue $\mathrm{CNO3}$, rescued photoreceptor 
viability and function in the genetically distinct $r d 1, r d 2$, and $r d 10$ animal models (Vighi, Trifunovic, et al. 2018). These results confirmed cGMP/PKG-signaling as a common target for the mutation-independent treatment of different RD-type diseases. Since then, several promising and potent $2^{\text {nd }}$ generation, novel cGMP analogues have been developed, but their biochemical properties and neuroprotective efficacy have not been examined thus far.

Here, we identified a novel cGMP analogue with strong photoreceptor-protective effects in retinal explants derived from $r d 1$ and $r d 10$ mice. We investigated the effects of this compound on retinal function in long-term organotypic retinal explant cultures derived from rd10 and wild-type (WT) mice, using micro-electrode arrays (MEAs). The recordings revealed a stronger photoreceptor response and, surprisingly, also increased retinal ganglion cell (RGC) activity in the treated samples. Increased RGC viability in treated specimens was confirmed by histological analysis. Together, these results give new insights into the properties of cGMP analogues and other neuronal cell types.

\section{Materials and Methods}

Animals. C3H Pde6b $b^{r d 1 / r d 1}$ (rd1), congenic C3H wild-type (C3H), C57BL/6J wild-type (C57) and

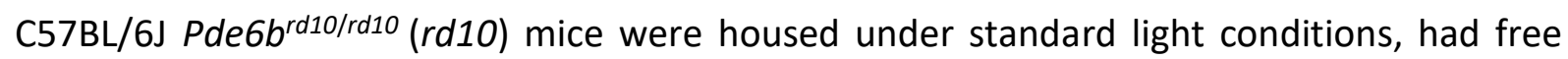
access to food and water, and were used irrespective of gender. All procedures were performed in accordance with the ARVO declaration for the use of animals in ophthalmic and vision research and the law on animal protection issued by the German Federal Government (Tierschutzgesetz) and were approved by the institutional animal welfare office of the University of Tübingen.

cGMP analogues synthesis. Synthesis of cyclic nucleotide analogues was performed by Biolog Life Science Institute GmbH \& Co. KG according to previously described methods (Vighi, Trifunovic, et al. 2018) (https://patentscope.wipo.int/search/en/detail.jsf?docld=WO201801 0965).

In vitro PKG activation/inhibition assay. FLAG-Strep-Strep-tagged human PKG 1 $\alpha$ (2-671) Wt, human PKG1ß (4-686) Wt and human PKG2 (1-762) Wt were expressed in HEK293T cells. Cells were transfected at $80 \%$ confluency in whole medium employing the transfection reagent polyethyleneimine (Polysciences Europe $\mathrm{GmbH}$, Germany). The cells were lysed using $50 \mathrm{mM}$ Tris- $\mathrm{HCl}$ (pH 7.3), $150 \mathrm{mM} \mathrm{NaCl}, 0.5 \mathrm{mM}$ TCEP, $0.4 \%$ Tween, protease and phosphatase 
inhibitors (Roche, Germany). For purification we employed Strep- Tactin ${ }^{\circledR}$ Superflow $^{\circledR}$ resin (IBA GmbH, Germany). We included an additional washing step with $366 \mathrm{mM} \mathrm{Na} 2 \mathrm{HPO}_{4}$, $134 \mathrm{mM} \mathrm{NaH}_{2} \mathrm{PO}_{4}(\mathrm{pH} 7.3)$ and $0.5 \mathrm{mM} \mathrm{TCEP}$ at room temperature to release any remaining nucleotides from the respective nucleotide binding pockets. Strep-tagged proteins were eluted with $200 \mathrm{mM}$ Tris- $\mathrm{HCl}$ (pH 8), $300 \mathrm{mM} \mathrm{NaCl}, 2 \mathrm{mM}$ EDTA and 5 mM desthiobiotin (IBA $\mathrm{GmbH}$, Germany) and subsequently stored at $4^{\circ} \mathrm{C}$ in $50 \mathrm{mM}$ Tris- $\mathrm{HCl}$ buffer ( $\mathrm{pH} 7.3$ ) containing $150 \mathrm{mM} \mathrm{NaCl}$ and $0.5 \mathrm{mM}$ TCEP.

PKG kinase activity was assayed in vitro using a coupled spectrophotometric assay originally described by Cook et al. (Cook et al. 1982) in a clear 384 well PS-MICROPLATE (Greiner BioOne, USA) in a CLARIOstar plate reader (BMG LABTECH, Germany). The final assay mixture contained 100 mM MOPS (pH 7.0), $10 \mathrm{mM} \mathrm{MgCl}$, 1 mM ATP, 1 mM phosphoenolpyruvate, $15.1 \mathrm{U} / \mathrm{ml}$ lactate dehydrogenase, $8.4 \mathrm{U} / \mathrm{ml}$ pyruvate kinase, $230 \mu \mathrm{M}$ reduced nicotinamide adenine dinucleotide, $0.1 \mathrm{mg} / \mathrm{ml} \mathrm{BSA}, \mathrm{mM} \beta$-mercaptoethanol and, as PKG substrate, $1 \mathrm{mM}$ VASPtide (RRKVSKQE; GeneCust, Luxembourg). PKG Activation was determined with cGMP (Supplemental Figure S1) and the cGMP analogues CN003, CN226, and CN238 (all Biolog Life Science Institute $\mathrm{GmbH} \&$ Co. KG, Germany) in dilution series ranging from $100 \mu \mathrm{M}$ to $5.1 \mathrm{nM}$. The kinase reaction was started with $5 \mathrm{nM}$ of the corresponding PKG isoform. Inhibition studies were performed by adding each PKG isoform supplemented with $2 \mu \mathrm{M}$ cGMP to the assay mix and the respective cGMP analogue in dilutions ranging from $100 \mu \mathrm{M}$ to $5.1 \mathrm{nM}$. One to three independent protein preparations were used for each assay, which in turn were performed in duplicates.

Organotypic retinal explant cultures. Organotypic retinal cultures derived from $\mathrm{C} 57, \mathrm{rd} 10$, rd1, and C3H animals, were prepared as previously described (Belhadj et al. 2020; Caffé et al. 2001) under sterile conditions. Post-natal day (P)5 rd1, P9 or P12 rd10 animals were sacrificed, the eyes rapidly enucleated and incubated in R16 retinal culture medium (07491252A; Gibco; Waltham, Massachusetts, USA) with 0.12\% proteinase K (21935025; ICN Biomedicals Inc., Costa Mesa, California, USA) for $15 \mathrm{~min}$ at $37^{\circ} \mathrm{C}$. Proteinase $\mathrm{K}$ activity was blocked by the addition of $20 \%$ foetal bovine serum (FCS) (F7524, Sigma) followed by rinsing in R16 medium. Afterwards, the anterior segment, lens, vitreous, sclera, and choroids were removed, while the RPE remained attached to the retina. The explant was cut into a four-wedged shape resembling a clover leaf and transferred to a culture membrane insert (3412; Corning Life Sciences) with the RPE facing the membrane. The membrane inserts were placed into six-well 
culture plates and incubated with complete R16 medium with supplements and free of serum and antibiotics (Belhadj et al. 2020), in a humidified incubator ( $5 \% \mathrm{CO} 2)$ at $37^{\circ} \mathrm{C}$. For the first $48 \mathrm{~h}$ the retinae were cultured with complete R16 medium without any treatment to allow adaptation to culture conditions. Afterwards, they were either exposed to different cGMP analogues (dissolved in water), each at [50 $\mu \mathrm{M}]$, or kept as untreated control. In both cases, medium was changed every second day with replacement of the full volume of the complete R16 medium, $1 \mathrm{~mL}$ per dish, with fresh medium. The culturing paradigm was from P5 (explantation) to P11 (end of culture) for $r d 1$. For the $r d 10$ model, two culturing paradigms were used: from P9 to either P17 or P19, and from P12 to P24. Culturing was stopped by 45 min fixation in $4 \%$ paraformaldehyde (PFA), cryoprotected with graded sucrose solutions containing 10, 20, and $30 \%$ sucrose and then embedded in Tissue-Tek O.C.T. compound (Sakura Finetek Europe, Alphena and enRijn, Netherlands). Tissue sections of $12 \mu \mathrm{m}$ were prepared using Thermo Scientific NX50 microtome (Thermo Scientific, Waltham, MA) and thaw-mounted onto Superfrost Plus glass slides (R. Langenbrinck, Emmendingen, Germany).

Histology. For retinal cross-sectioning preparation, the eyes were marked nasally, and cornea, iris, lens, and vitreous were carefully removed. The eyecups were fixed in $4 \%$ PFA, cryoprotected in sucrose, and sectioned as above.

TUNEL assay. The various CGMP analogues were tested for their effect on photoreceptor cell death, using terminal deoxynucleotidyl transferase dUTP nick end labeling (TUNEL) assay (Loo 2011) (Sigma-Aldrich in situ Cell Death Detection Kit, 11684795910, red fluorescence). DAPI contained in the mounting medium (Vectashield antifade mounting medium with DAPI; Vector Laboratories, Burlingame, CA, USA) was used as nuclear counterstain.

Immunofluorescence. Immunostaining with primary antibody against rabbit RBPMS (1:500; Abcam, Cambridge, UK) was performed on $12 \mu \mathrm{m}$ thick retinal explants cryosections by incubating at $4{ }^{\circ} \mathrm{C}$ overnight. Alexa Fluor 488 antibody was used as secondary antibody. Sections were mounted with Vectashield medium containing 4',6-diamidino-2-phenylindole (DAPI, Vector).

Microscopy and image processing. Images were captured using 7 Z-stacks with maximum intensity projection (MIP) on a Zeiss Axio Imager Z1 ApoTome Microscope MRm digital camera (Zeiss, Oberkochen, Germany) with a 20x APOCHROMAT objective. For more details about the characteristics of the filter sets for the fluorophores used see Table 1. For the quantifications 
of positively labelled cells, pictures were captured on at least six different areas of the retinal explant for at least four different animals for each genotype. Adobe Photoshop (CS5Adobe Systems Incorporated, San Jose, CA) was used for image processing.

\begin{tabular}{|l|c|c|}
\hline Fluorophore & exc. max. / em. max. (nm) & exc. filter / em. filter (nm) \\
\hline TMR red & $540 / 580$ & $538-562 / 570-640$ \\
\hline AF488 & $495 / 519$ & $450-490 / 500-550$ \\
\hline
\end{tabular}

Table 1: Fluorophores and microscope filters. Excitation (exc.) and emission (em.) characteristics of the TMR red and AF488 and of the microscope filter sets used to visualize them.

Ex-vivo retinal function test. Prior to recording organotypic retinal cultures were kept dark for at least $12 \mathrm{~h}$, further manipulations were performed under dim red-light. The retinas were divided into two equal halves, one of which was placed immediately on the electrode field of the recording chamber and kept in the dark. The second retinal half was used for histological preparation and immunofluorescence (see above). Two recordings were obtained from locations within the central retinal half. Retinal function tests were performed in R16 medium, and the recording chamber temperature was set to $37^{\circ} \mathrm{C}$. To record the light-evoked retinal responses, a micro-electrode array system (MEA; USB-MEA60-Up-BC-System-E, Multi Channel Systems; MCS; Reutlingen, Germany), equipped with HexaMEA 40/10iR-ITO-pr (60 electrodes = 59 recording and one reference electrode) was employed. The recordings were performed at $25.000 \mathrm{~Hz}$ sampling rate to collect unfiltered raw data. The trigger synchronized operation of the light stimulation (LEDD1B T-Cube, Thorlabs, Bergkirchen, Germany) and MEA-recording were controlled by a dedicated protocol implemented within the MC-Rack software ( $v$ 4.6.2, MCS) and the digital I/O - box (MCS). The light stimulation (white light LED, $2350 \mathrm{~mW}$, MCWHD3, Thorlabs), guided by fiber-optic and optics, was applied from beneath the transparent glass MEA: five full field flashes of $500 \mathrm{~ms}$ duration with $20 \mathrm{~s}$ intervals. A spectrometer USB4000-UV-VIS-ES (Ocean Optics, Ostfildern, Germany) was employed to calibrate the intensity of the applied light stimulation $\left(1,33 E+14\right.$ photons $\left./ \mathrm{cm}^{2} / \mathrm{sec}\right)$. For the analysis of the electrophysiology data, custom-developed scripts (MATLAB, The MathWorks, Natick, MA, USA) were used, if not indicated otherwise. MEA-recording files were filtered employing the Butterworth $2^{\text {nd }}$-order (MC-Rack, MC) to extract retinal ganglion cell spikes (high pass $200 \mathrm{~Hz}$ ) and field potentials (bandpass $2-40 \mathrm{~Hz}$ ). The field potentials recorded by 
bioRxiv preprint doi: https://doi.org/10.1101/2021.08.05.455191; this version posted September 7, 2021. The copyright holder for this preprint (which was not certified by peer review) is the author/funder, who has granted bioRxiv a license to display the preprint in perpetuity. It is made available under aCC-BY-NC-ND 4.0 International license.

Tolone et al., 2021 PKG inhibition and retinal neuroprotection.

the MEA system are referred as micro-electroretinogram ( $\mu \mathrm{ERG}$ ), and largely correspond to the human electroretinogram (ERG) as described by (Stett et al. 2003). The filtered data were converted to *.hdf files by MC DataManager (v1.6.1.0). Further data processing was performed in MATLAB (spike and field potential detection) as previously described (Haq et al. 2018; Haq, Dietter, and Zrenner 2018).

Statistics. 1) Analysis of PKG activation/inhibition assay: data were analyzed using GraphPad Prism 8.0.1 (GraphPad Software, Inc, La Jolla, CA, USA). Activation ( $\mathrm{K}_{\text {act }}$ ) and inhibition (IC $\left.\mathrm{C}_{50}\right)$ data are presented as mean \pm standard deviation (SD); $n=$ at least 3 except CN226 ( $n=2)$.

2) Analysis of retinal cell death: The total number of TUNEL positive cells in the defined area of the outer nuclear layer (ONL) were estimated by dividing the ONL area by the average area occupied by a cell (i.e., cell size). The number of positively labelled cells in the ONL was counted manually on pictures captured on at least six different areas of the retinal explant for at least four different animals for each genotype. Only cells showing a strong staining of the photoreceptor nuclei were considered as positively labelled. Values obtained are given as fraction of total cell number in ONL (i.e., as percentage) and expressed as mean \pm SD.

3) Analysis of ganglion cell survival: An area of $1 \mathrm{~mm}^{2}$ was divided by the product of the length of counting and the section's thickness $(12 \mu \mathrm{m})$. The number of RBPMS positive cells was counted manually. For statistical analysis in both 1) and 2) a one-way ANOVA testing followed by the Dunnett's multiple comparison test as implemented in Prism 8 for Windows (GraphPad Software) was conducted.

4) Analysis of electrophysiology data: For the quantification of the PKG inhibitor effects on retinal light sensitivity, the photoreceptor ( $\mu \mathrm{ERG}$ ) and ganglion cell (spikes) responses were considered: (1) Light responsiveness: This is represented by the percentage of light-dependent $\mu E R G$ detecting electrodes, to estimate the retinal light-sensitivity and to indirectly infer the density of functional photoreceptors in a given electrodes recording field. Note that a single MEA electrode captures the integrated signal of multiple photoreceptors within the recording field. The 59 MEA electrodes with $40 \mu \mathrm{m}$ spacing together span an overall recording field of $340 \times 280 \mu \mathrm{m}$, allowing to estimate the retinal light-sensitivity at 59 different positions. A $\mu E R G$ response upon light stimulation was counted as light-responsive if exceeding the respective threshold (response amplitude 1.75-fold $\geq$ calculated average of $500 \mathrm{~ms}$ control pre-stimulus baseline). (2) Deflection of the negative wave of the $\mu E R G$ : This measure reflects 
the strength of the light-evoked photoreceptor response - its hyperpolarization, equivalent to the a-wave in a conventional ERG - indicated by the initial negative deflection of the $\mu \mathrm{ERG}$ (Figure 3 C, arrow). (3) Spike responses were accounted as light-stimulus correlated, if the post stimulus activity (average of 6 bin counts: 500 ms light duration and 100 ms post stimulation, 100 ms binning) exceeded the pre-stimulus activity (threshold: average of 5 bin counts prestimulus; $500 \mathrm{~ms}$ and $100 \mathrm{~ms}$ binning). Activity maps were generated to reflect the $\mu \mathrm{ERG}$ recordings in their spatial context. Each pixel corresponds to a recording electrode of a MEA (center-center) and its surrounding recording area. The color encodes the negative deflection of the recorded $\mu \mathrm{ERG}$ (-5 $\mu \mathrm{V}$ binning). For statistical analysis of the $r d 10$ data, one-way ANOVA followed by the Dunnett's multiple comparison test was applied and for the WT dataset the Wilcoxon-Mann-Whitney test was utilized (MATLAB, The MathWorks).

\section{Results}

\section{Novel PKG inhibitors protect $r d 1$ photoreceptors.}

Cyclic nucleotide (CN) analogues of cGMP (Figure 1) were previously generated in the context of the EU project DRUGSFORD (HEALTH-F2-2012-304963) and amongst others tested for their protective effects in primary rod-like cells. Here, we selected cGMP analogues that shared structural similarities with the retinoprotective compounds CN003 and CNO04 (Vighi, Trifunovic, et al. 2018), which served as references. Structure wise all compounds feature a cGMPS backbone containing a sulfur-modified phosphate function with Rp-configuration, which confers PKG inhibitory properties (Zhao et al. 1997). Further modifications were introduced on the nucleobase moiety at positions $8\left(R_{1}\right)$ and $1, N^{2}\left(R_{2}, R_{3}\right)$. Therein the reference compounds contain a so-called PET-group ( $\beta$-phenyl- $1, N^{2}$ - etheno) at $1, N^{2}$ while this group is either lacking (CN226), substituted with an additional methyl-group (CN238), or replaced through the heteroaromatic furan ring (CNO07). At position 8 the residue in CN226 contains a phenyl ring as present in reference compound CNOO4, however deviating from CN004 where a different, slightly larger linker has been introduced and said phenyl ring is unsubstituted. CNOO7 and CN238, in turn, share the same bromide function as in the reference compound CN003. A compound concentration of $50 \mu \mathrm{M}$ was used, based on previous in vitro results obtained with the reference compounds (Vighi, Trifunovic, et al. 2018). 


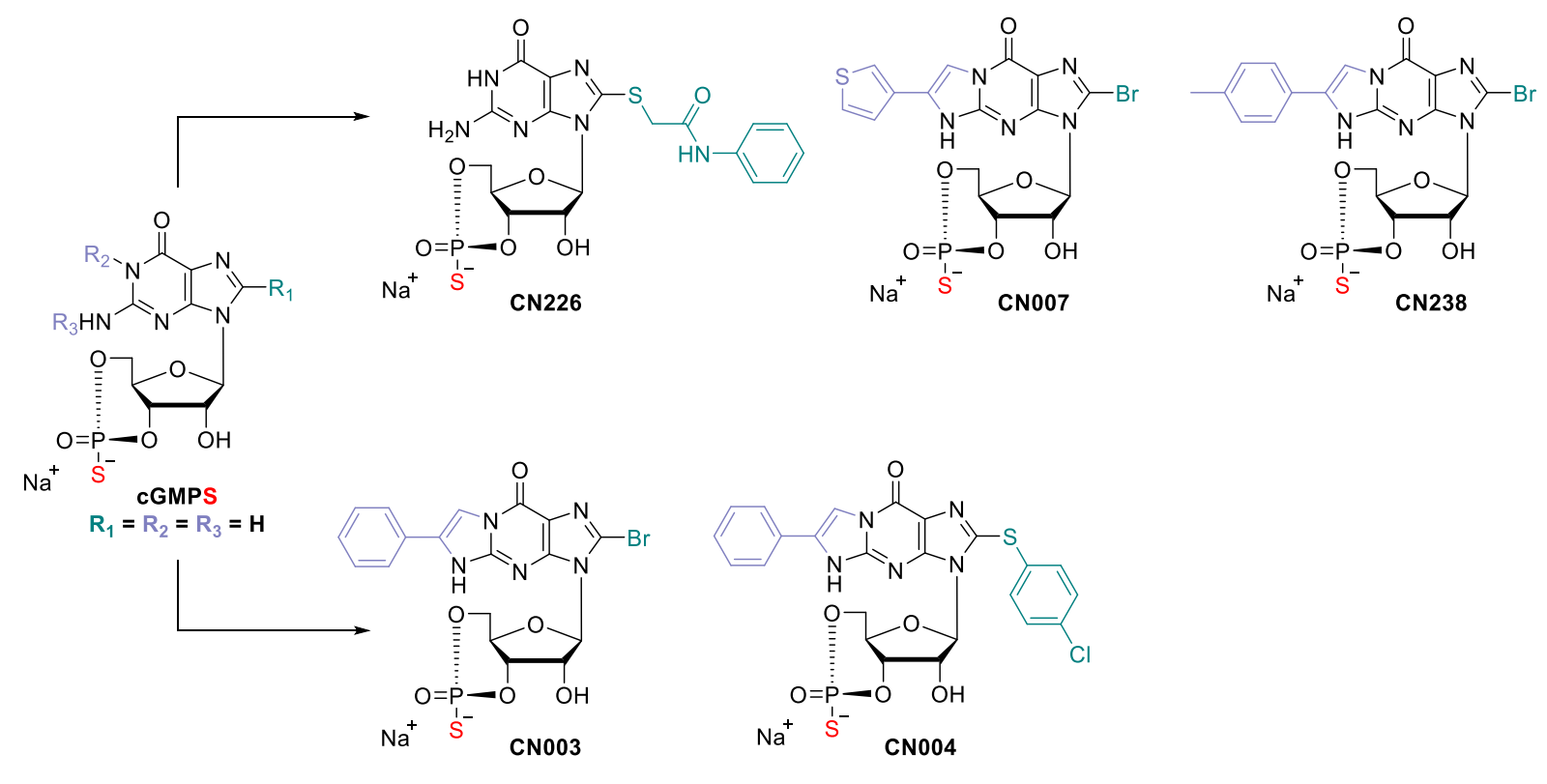

Figure 1: Structures of tested novel cyclic nucleotide analogues of cGMP and reference compounds. CN003: 8- Bromo- $\beta$ - phenyl- 1, $\mathrm{N}^{2}$ - ethenoguanosine- 3', 5'- cyclic monophosphorothioate, Rp- isomer (Rp-8-Br-PET-cGMPS); CN004: 8- (4- Chlorophenylthio)$\beta$ - phenyl- 1, N2- ethenoguanosine- 3', 5'- cyclic monophosphorothioate, Rp- isomer (Rp-8pCPT-PET-cGMPS); CN007: 8- Bromo- (3- thiophen- yl- 1, $\mathrm{N}^{2}$ - etheno)guanosine- 3', 5'- cyclic monophosphorothioate, Rp- isomer (Rp-8-Br-(3-Tp)ET-cGMPS); CN226: 8Phenylamidomethylthioguanosine- 3', 5'- cyclic monophosphorothioate, Rp- isomer (Rp-8PAmdMT-cGMPS); CN238: 8- Bromo- (4- methyl- $\beta$ - phenyl- $1, \mathrm{~N}^{2}$ - etheno) guanosine- $3^{\prime}, 5^{\prime}-$ cyclic monophosphorothioate, Rp- isomer (Rp-8-Br-pMe-PET-cGMPS).

We initially tested the cGMP analogues on organotypic retinal explant cultures derived from $r d 1$ mouse. The loss of function of PDE6 in rd1 results in primary loss of rods already during development, with a peak around P13 and almost complete loss at P18 (Sahaboglu et al. 2013). Therefore, in $r d 1$ explants treatment with cGMP analogues started at P7 and ended at P11, i.e., at the onset of manifest retinal degeneration (Sancho-Pelluz et al. 2008), a timepoint well suited for establishing possible protective effects.

As a readout of the effects of cGMP analogue treatments, we performed TUNEL assays on sections obtained from treated and non-treated (NT) specimens (Figure 2A). We considered any reduction in TUNEL positivity in the ONL indicative of a decrease in photoreceptor degeneration. When compared to $r d 1$ NT (100\%), retinae treated with the compounds CN007, CN226, and CN238 (NT = $7.03 \pm 1.61 ;$ CN007 = $5.04 \pm 1.26 ; C N 226=6.18 \pm 0.66 ; C N 238=4.39$ \pm 0.50 ) showed $\mathrm{a} \approx 32 \%, \approx 17 \%$ and $\approx 40 \%$ reduction of TUNEL positive cells, respectively. The reference compounds $\mathrm{CNO03}$ and $\mathrm{CNOO4}$ ( $\mathrm{CNOO3}=4.79 \pm 0.50$; $\mathrm{CNOO4}=4.49 \pm 0.45$ ) confirmed their previously seen protective effects (Vighi, Trifunovic, et al. 2018) (Figure 2B). 
bioRxiv preprint doi: https://doi.org/10.1101/2021.08.05.455191; this version posted September 7, 2021. The copyright holder for this preprint (which was not certified by peer review) is the author/funder, who has granted bioRxiv a license to display the preprint in perpetuity. It is made available under aCC-BY-NC-ND 4.0 International license.

Tolone et al., 2021

PKG inhibition and retinal neuroprotection.

We then assessed the efficacy of the most promising compound CN238 to inhibit the PKG isoforms PKG1 $\alpha, P K G 1 \beta$, and PKG2. As references in this characterization, we included the previous lead compound CN003, as well as the compound CN226 as a "negative control" since it had not shown protection in $r d 1$ retinal explants (Supplemental Figure S2). This analysis indicated a slightly increased potency of CN238 towards PKG1 and PKG2 and furthermore revealed this compound to have partial agonistic effects on PKG1 $\alpha$ at high concentrations. This suggests that CN238 is in fact modulating or dampening PKG1 $\alpha$ activity rather than completely blocking it. On the other hand, analysis of the efficacy of CN226 showed that it was only weakly inhibiting PKG1 $\beta$ and PKG2, and that it did in fact activate PKG1 $\alpha$, in line with our results on $r d 1$ retinal explants (cf. Figure 1 ).
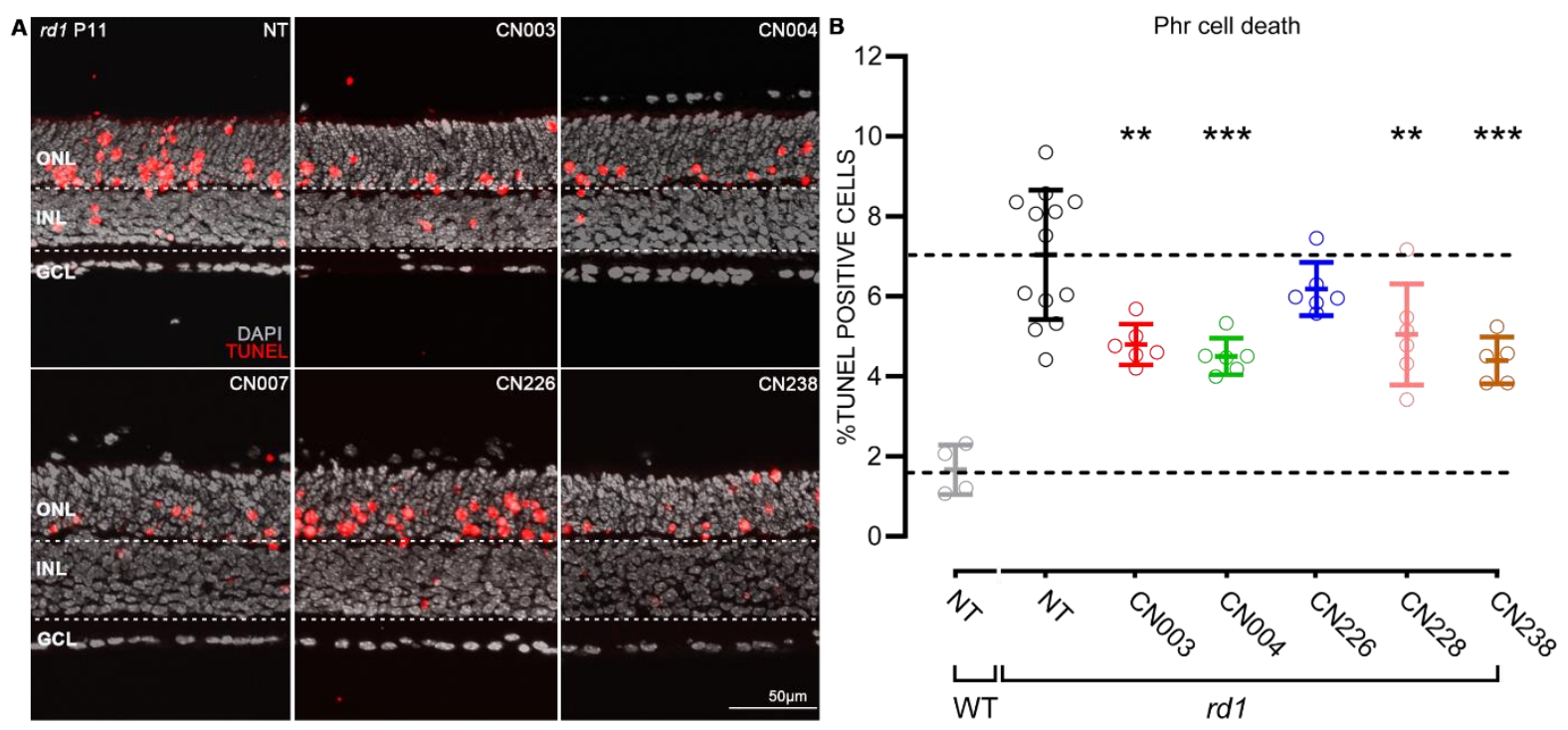

Figure 2: Retinoprotective effects of novel PKG inhibitors on rd1 P11 organotypic retinal explant cultures. (A) Post-natal (P) day $11 \mathrm{rd} 1$ retinal explant cultures treated for four days with $50 \mu \mathrm{M}$ of different cGMP analogues. TUNEL assay (red) indicated dying cells, DAPI (grey) was used as nuclear counterstain. (B) Quantification of TUNEL positive cells in the outer nuclear layer (ONL) of sections from A. Cell death rate in NT WT retina shown for comparison. A cell death rate lower than in the non-treated (NT) $r d 1$ retina was interpreted as evidence for photoreceptor protection. Compounds $\mathrm{CNOO3}$ and $\mathrm{CNOO4}$ had previously been established as photoreceptor protective (Vighi, Trifunovic, et al. 2018) and were used for reference. Testing was performed on $n=4$ to 12 different retinae from different animals. Error bars: mean with SD. Statistical analysis was performed using one-way ANOVA followed by the Dunnett's multiple comparison test; significance levels were: ${ }^{* *} P \leq 0.01,{ }^{* * *} P \leq 0.001$. INL $=$ inner nuclear layer, GCL = ganglion cell layer. 


\section{PKG inhibition preserves viability and function of photoreceptors in rd10 retinal explants.}

To assess the validity of these results across different animal models, we further tested the most promising compound $\mathrm{CN} 238$ and the reference compound $\mathrm{CNO} 03$ on organotypic retinal explant cultures derived from $r d 10$ mice. As in $r d 1$ animals, the $r d 10$ mutation affects the gene encoding for the $\beta$-subunit of rod PDE6, however, in rd10 photoreceptors the PDE6 enzyme retains some residual activity, delaying the onset of photoreceptor degeneration until P18 (Arango-Gonzalez et al. 2014; Power et al. 2020). Hence, the culturing and treatment paradigms were adjusted accordingly, and $r d 10$ retinae were cultured from either P9 to P17 or P19, or from P12 till P24. As in the rd1 situation, and to allow the retinal explant to adapt to culture conditions, drug treatments were begun two days after explantation.

While the TUNEL assay gives a count of the number of dying cells at a given age, the number of mutant photoreceptors still surviving compared to the number of photoreceptors in an agematched NT retina, gives the integral of cells preserved until a given time-point (Figure 3A). We therefore analyzed the number of photoreceptor rows to assess the long-term effects of the compounds. When $\mathrm{CNOO3}$ and $\mathrm{CN} 238$ were tested on rd10 retina in treatments reaching until P17 or P19 there was no statistically significant rescue, likely because of the comparatively late onset of $r d 10$ degeneration around P18. At P24, however, a clear and highly significant rescue of $r d 10$ photoreceptors was observed, with an increase in the photoreceptor row counts of $\approx 55 \%$ and $\approx 46 \%$ in samples treated with CN003 and CN238 (NT = $3.86 \pm 1.01$; CN003 = 6.01 $\pm 0.87 ;$ CN238 $=5.64 \pm 0.32$ ), respectively (Figure 3B). 

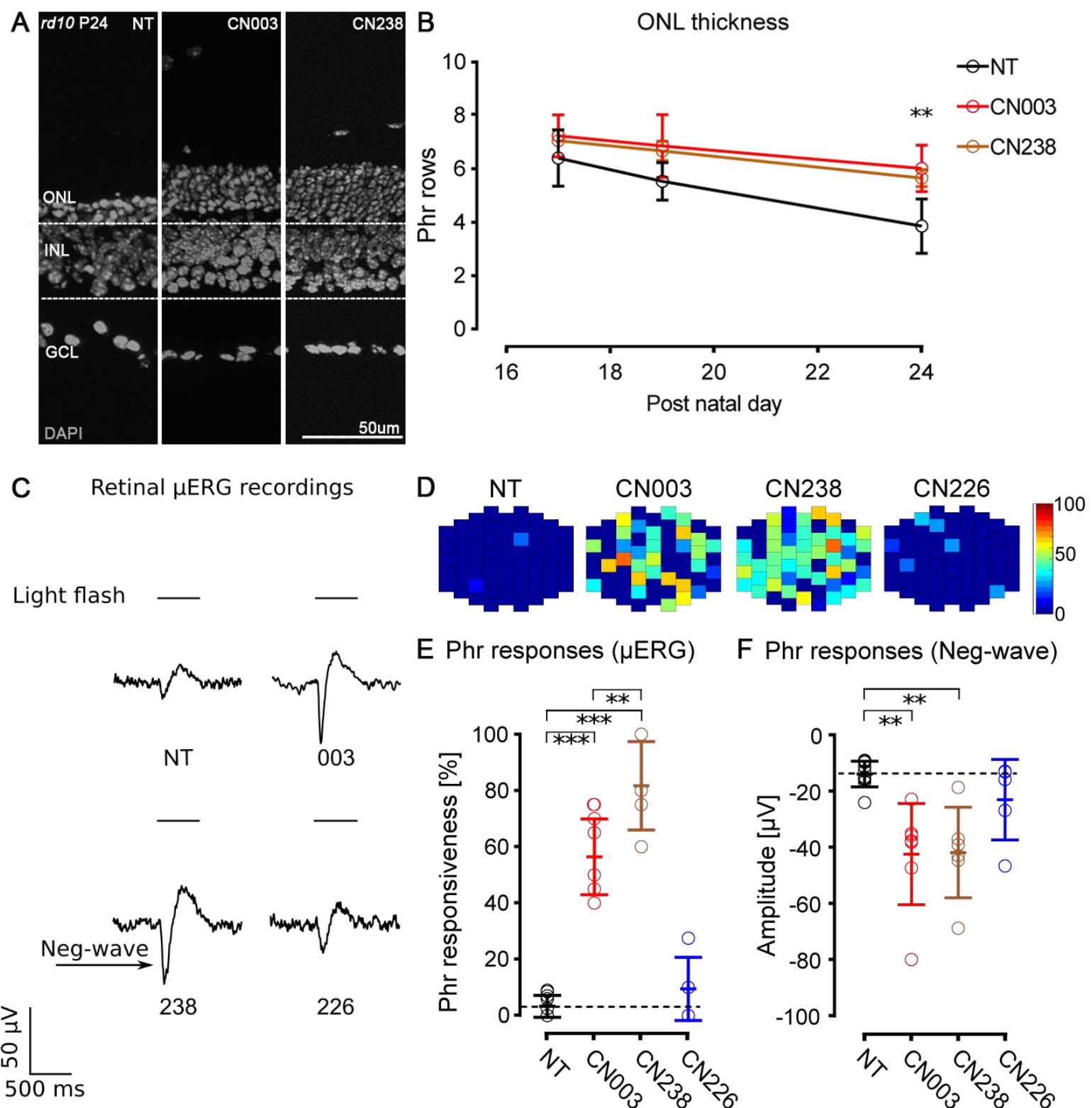

E Phr responses ( $\mu$ ERG) F Phr responses (Neg-wave)
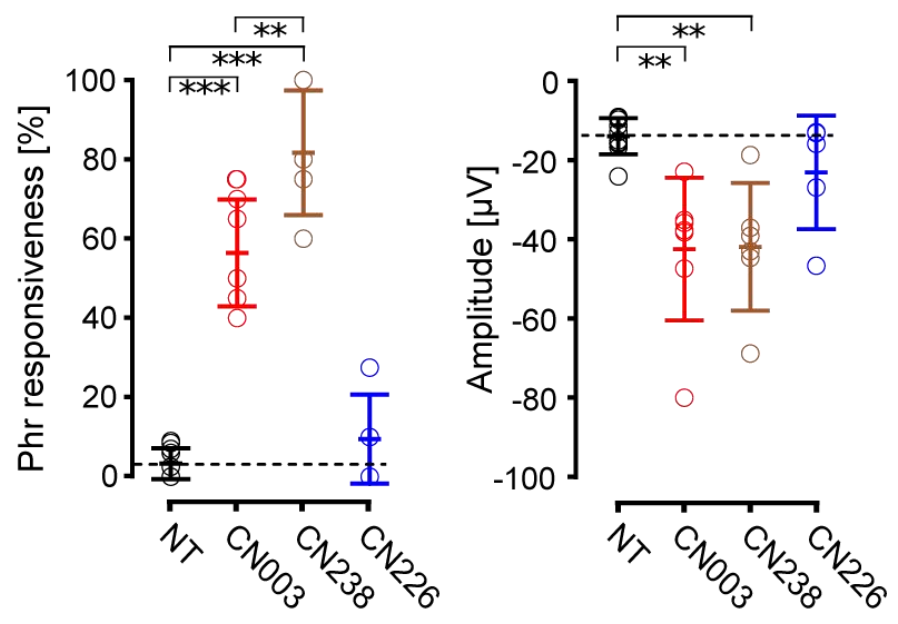

Figure 3: CN238 preserves photoreceptor viability and function in $r d 10$ retina: Organotypic retinal explant cultures derived from $r d 10$ mice were treated with cGMP analogues at $50 \mu \mathrm{M}$ concentration. (A) Representative sections of post-natal (P) day 24 rd10 retinal cultures treated or non-treated (NT) and stained with DAPI (grey). (B) rd10 retinal explants were treated for varying times until either P17, P19, or P24, and photoreceptor rows were quantified. Row counts above NT were interpreted as evidence for photoreceptor protection. Testing was performed on $n=5$ different retinae from different animals. (C) Representative $\mu E R G$ traces of NT and treated $r d 10$ retinal explants. The single-electrode data represents the integrated signal of multiple photoreceptors above a given electrodes recording field. The strength of the light-evoked photoreceptor hyperpolarization is indicated by the initial negative deflection (neg.-wave) of the $\mu E R G$ (arrow). (D) Activity map of representative $\mu E R G$ recordings across the 59 electrodes of the MEA, indicating the reactivity to light in spatial context. Each pixel corresponds to a recording electrode and the color from blue to red (i.e., 
0 to 100 ) encodes the increasing intensity of the negative deflection of the recorded $\mu E R G$. (E) Quantification of retinal light responsiveness as percentage of MEA electrodes displaying $\mu E R G$ negative deflections $\geq 1.75$-fold average baseline. Testing was performed on $n=5$ different retinae from different animals. (F) Average amplitudes of negative deflection in $\mu E R G$ recordings. Error bars: mean with SD. Statistical testing: one-way ANOVA with Dunnett's multiple comparison test; significance level: ${ }^{* *} P \leq 0.01$. INL = inner nuclear layer, $\mathrm{Phr}=$ photoreceptor, $\mu \mathrm{ERG}=$ micro-electroretinogram, $\mathrm{GCL}=$ ganglion cell layer, $\mathrm{MEA}=$ microelectrode array.

To determine whether the increased photoreceptor survival seen with PKG inhibition also translated into improved retinal function, we performed electrophysiological recordings of rd10 retinal explants employing a micro-electrode array (MEA) system and white light LED stimulator to apply $500 \mathrm{~ms}$ full-field light flashes. The method allowed for the selective assessment of photoreceptor functionality by recording light-elicited microelectroretinograms ( $\mu \mathrm{ERG})$. In this analysis, we included the compound that performed best in the retinal cell death assay, CN238 (cf. Figure 2), along with the previous lead compound CN003 and CN226, which here served as additional "negative" control.

As shown in representative $\mu E R G$ traces (Figure $3 C$ ), after 12 days of in vitro culture the PKG inhibitors CNO03 and CN238 strongly increased the amplitudes of light-induced retinal responses, when compared to NT and CN226. In particular, the initial negative deflection of the $\mu \mathrm{ERG}$ (Figure $3 \mathrm{C}$, arrow), indicated a light-induced hyperpolarization of photoreceptors (i.e., the a-wave in a conventional ERG) and thus their functional preservation with drug treatment. For each retina, recordings were obtained from two different areas located dorsally and ventrally from the center and averaged (each recording area: $340 \times 280 \mu \mathrm{m}$; 59 electrodes at $40 \mu \mathrm{m}$ spacing). Since each of the MEA electrode captured the integrated signal of multiple photoreceptors within an electrode's recording range, the total span of the MEA recording field allowed an estimation of the overall light-sensitivity of a given retinal explant. We considered a light-induced negative $\mu E R G$ deflection exceeding respective threshold (1.75fold $\geq$ calculated average control baseline) to indicate light-responsiveness and drew activity maps for the MEA electrodes showing light responses (Figure 3D). We then expressed the number of electrodes showing such a response as percent of the total (Figure $3 \mathrm{E}$ ). This analysis revealed that NT retina, and CN226 treated retina, displayed almost no response to light (NT $=3.3 \pm 3.9 \%$; CN226 = $9.5 \pm 11.2 \%$ ), while CN003 (56.4 $\pm 13.5 \%$ ), and even more so CN238 $(81.7 \pm 15.7 \%)$, strongly and significantly increased light responsiveness of treated retinal 
explants (Figure 3E). In other words, treatment with CN003 or CN238 dramatically increased the retinal area responding to light by $\approx 17$ - or $\approx 25$ - times, respectively, when compared to NT. Compared to CNO03, CN238 increased retinal responsiveness by yet another $\approx 45 \%$, suggesting a further improvement of photoreceptor function and/or the density of the photoreceptors activating a given MEA electrode.

As an additional measure of photoreceptor functionality, we quantified the amplitudes of the initial negative $\mu E R G$ deflection, as a measure for the light-induced photoreceptor hyperpolarization. When compared to NT, retinal explants treated with $\mathrm{CN} 003$ or $\mathrm{CN} 238$ on average displayed a significantly stronger hyperpolarization response to light (NT: $-13.6 \pm 4.6$ $\mu \mathrm{V}$; CN003: $-42.1 \pm 18.0 \mu \mathrm{V}$; CN238: $-41.5 \pm 16.1 \mu \mathrm{V})$. In contrast, CN226 treatment had only a minor effect on response amplitudes $(-22.7 \pm 14.3 \mu \mathrm{V})$ and/or the density of the photoreceptors activating a given MEA electrode (Figure 3F).

Taken together, we have established that the PKG inhibitors $\mathrm{CNO03}$ and $\mathrm{CN} 238$ preserved not only the viability of photoreceptor cells but also their functionality. In these comparisons the effects of the novel compound CN238 were at least equal, if not superior, to the previous lead compound CNOO3.

\section{CN238 prevents axotomy-induced degeneration of retinal ganglion cells (RGCs)}

The MEA recordings of treated $r d 10$ retinal explants revealed another feature that was entirely unexpected: during the retinal explantation procedure the optic nerve is transected, and this axotomy leads to rapid degeneration and loss of most RGCs within 4-7 days of in vitro culture (Alarautalahti et al. 2019; Berkelaar et al. 1994; Osborne et al. 2016). Accordingly, in NT rd10 retinal explants, cultured for 12 days in vitro, RGC spiking activity was virtually extinguished, and this was also true for CN226 treated explants. Yet, explants treated with CN003 or CN238 showed a very remarkable preservation of light-stimulus correlated RGC spiking activity (Figure $4 \mathrm{~A}$ ).

Similar to the analysis shown above for photoreceptor responses ( $\mu \mathrm{ERG}$ ), we assessed the light responsiveness of RGCs across the entire surface of the MEA chip, from two different central recording areas. Here, light-induced RGC spike responses were considered to be stimulus correlated, if post stimulus activity (600 ms) exceeded the average of pre stimulus activity (500 ms, $100 \mathrm{~ms}$ bin, see methods for details). While RGC light responsiveness in NT and CN226 treated cultures was nearly absent (NT = 3.3 $\pm 4.9 \%$; CN226 = $1.5 \pm 1.4 \%$ ), it was 
strongly and highly significantly increased in CN003 and CN238 treated retina (CN003 = $53.6 \pm$ $15.5 \%$; CN238: $49.2 \pm 8.8 \%$ ) (Figure 4B). Thus, in comparison to NT the area of the retina showing light responses at the RGC level was $\approx 16$ - or $\approx 15$-times larger after CN003 or CN238 treatment, respectively.

We then used the very same retinal explants from which the MEA recordings were obtained for a histological workup, to assess the survival and physical presence of RGCs. To this end, we employed labelling for the RNA-binding-protein-with-multiple-splicing (RBPMS), a protein that is expressed in about 60\% of RGCs (Rodriguez, de Sevilla Müller, and Brecha 2014) (Figure 4C). The quantification of RBPMS positive cells in the four experimental groups yielded very low RGC counts in NT and CN226 treated retina (NT = $134.1 \pm 117.3 \mathrm{~mm}^{2} ; \mathrm{CN} 226=184.1 \pm 81.06$ $\mathrm{mm}^{2}$ ), while CNO03 and CN238 treated explants displayed significantly larger numbers of RGCs (CNOO3 $=514.4 \pm 187.7 \mathrm{~mm}^{2} ; \mathrm{CN} 238=460.3 \pm 153.6 \mathrm{~mm}^{2}$ ). Compared to NT, retinal explants treated with $\mathrm{CNOO3}$ and $\mathrm{CN} 238$ showed 3.8- and 3.4-fold higher numbers of RBPMS positive cells, respectively. In line with the previous experiments, the compound CN226 did not preserve the viability of RGCs (Figure 4D). 
bioRxiv preprint doi: https://doi.org/10.1101/2021.08.05.455191; this version posted September 7, 2021. The copyright holder for this preprint (which was not certified by peer review) is the author/funder, who has granted bioRxiv a license to display the preprint in perpetuity. It is made available under aCC-BY-NC-ND 4.0 International license.
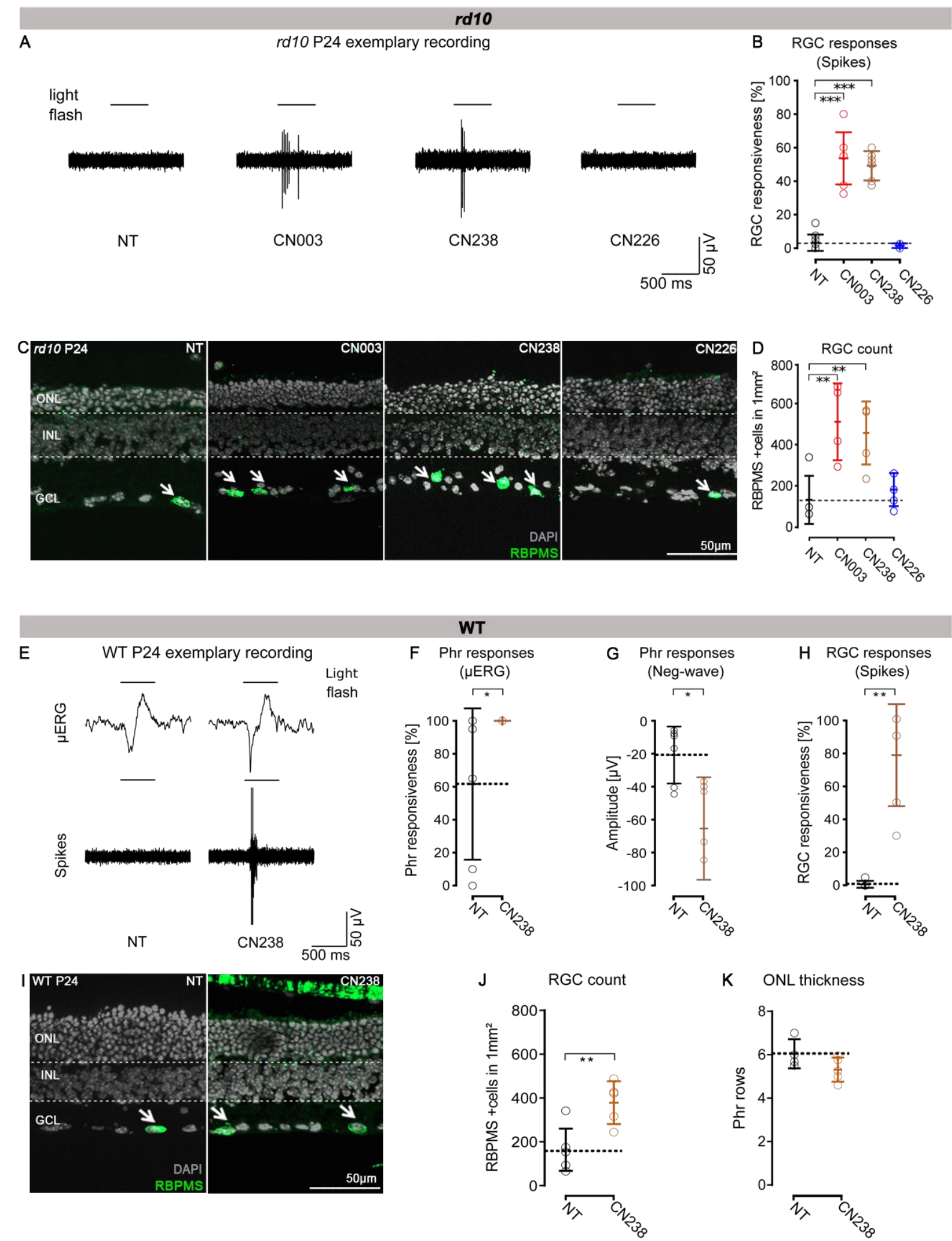

H RGC responses
(Spikes)

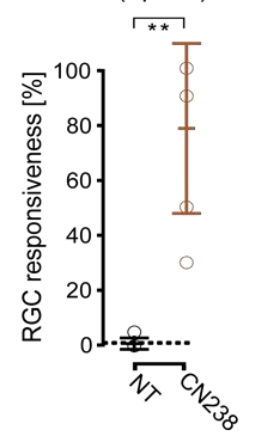

K ONL thickness

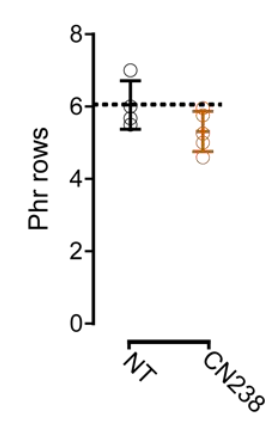

Figure 4: CN238 improves RGC viability and function in rd10 and WT retinal explant cultures.

(A) Representative light-correlated RGC spike MEA recordings performed on P24 rd10 retinal explants. $r d 10$ retinas were treated from post-natal day $(\mathrm{P}) 14$ to $\mathrm{P} 24$ with $50 \mu \mathrm{M}$ of $\mathrm{CNO03}$, CN238, or CN226, and compared to non-treated (NT) retinal explants. (B) Quantification of light-evoked rd10 RGC activity in NT and treated explants (percentage of MEA electrodes 
detecting light-correlated spike-activity). (C) Sections derived from recorded $r d 10$ retinal explant cultures were stained with DAPI (grey) and RBPMS (green). (D) Quantification of RBPMS positive cells in $r d 10$ P24 retinal explant sections. (E) Representative light-correlated RGC spike recordings of WT P24 retinal explants treated from P14 to P24 with $50 \mu \mathrm{M} \mathrm{CN} 238$ and compared to NT. (F-H) Quantification of light-stimulus evoked retinal activity in NT and treated WT explants: (F) light responsiveness (percentage of MEA electrodes detecting lightcorrelated $\mu E R G$ activity). (G) Quantification of negative $\mu E R G$ amplitudes. (H) RGC activity expressed as percentage of MEA electrodes detecting light-correlated spike-activity. (I) Sections derived from recorded WT P24 retinal explant cultures stained with DAPI (grey) and RBPMS (green). (J) Quantification of RBPMS positive cells in WT P24 retinal explants sections NT or treated with CN238. (K) Quantification of photoreceptor rows in NT and CN238 treated WT explants. Error bars indicate SD; statistical analysis in B, D: one-way ANOVA followed by Dunnett's multiple comparison test; in F, G, H: unpaired Student's $t$-test; levels of significance: $* P \leq 0.05, * * P \leq 0.01, * * * P \leq 0.001$. ONL = outer nuclear layer, $\mathrm{INL}=$ inner nuclear layer, $\mathrm{Phr}=$ photoreceptor, $\mu \mathrm{ERG}=$ micro-electroretinogram, $\mathrm{GCL}=$ ganglion cell layer, $\mathrm{MEA}=$ microelectrode array.

The magnitude of the rescue effect on axotomized RGCs raised the question whether this was a direct effect on RGCs or whether perhaps the preservation of $r d 10$ photoreceptors had indirectly enhanced the survival of $r d 10$ RGCs. To address this question, we extended our investigation to WT retinal explants, cultured for 12 days in vitro, from P12 to P24, treated or not with CN238.

The light-induced hyperpolarization response in the $\mu$ ERG obtained from P24 WT explants did not seem to differ between NT and CN238 treated retina, however, the RGC spiking activity was virtually absent in NT, but present after CN238 treatment (Figure 4E). The overall photoreceptor activity was obviously higher in WT than rd10 explants, yet even in the WT situation CN238 treatment improved light responsiveness somewhat (NT = $61.7 \pm 45.9 \%$; CN238 $=98.9 \pm 2.6 \%$ ) (Figure F). In line with this, the light-response amplitude in terms of negative deflection of the $\mu \mathrm{ERG}$ was also increased in CN238 treated explants compared to NT (NT = -26.2 $\pm 20.5 \mu \mathrm{V} ;$ CN238 = -79.0 $\pm 36.9 \mu \mathrm{V}$ ) (Figure G). The most striking effect of CN238 on WT retina was, however, observed at the level of RGC light-responsiveness: While NT retina showed nearly no light correlated activity, CN238 treatment largely preserved RGC light-induced spiking activity across the whole retinal explant (NT = $0.8 \pm 2.0 \%$; CN238 $=78.3$ $\pm 30.6 \%$ ) (Figure $4 \mathrm{H}$ ). In relative terms CN238 had thus increased WT RGC functionality by a striking $\approx 95$-times. 
Further confirmation came from an investigation of WT RGC viability with RBPMS staining, which showed RGCs survival 2.3-fold higher in retinae treated with CN238 (NT = $163.9 \pm 96.4$ $\mathrm{mm}^{2} ; \mathrm{CN} 238=379.3 \pm 97.7 \mathrm{~mm}^{2}$ ) (Figure 4J). In addition, we assessed photoreceptor survival in NT and CN238 treated samples, without detecting significant photoreceptor loss (Figure $4 K)$.

These findings thus confirmed the formidable capacity of CN238 to preserve RGC activity despite the axotomy caused by the explantation procedure. This data also demonstrated that the RGC protection was independent of photoreceptor degeneration.

\section{Discussion}

The excessive accumulation of cGMP in photoreceptors has long since been established as a trigger for the loss of photoreceptors in rare, RD-type diseases (Farber and Lolley 1974; Lolley et al. 1977). Here, using the novel inhibitory cGMP-analogue CN238, we validate PKG as the critical effector of cGMP-dependent cell death. Unexpectedly, the protective effect of PKG inhibition extended beyond photoreceptors to axotomized retinal ganglion cells, neurons whose degeneration is underlying common retinal diseases such as glaucoma and diabetic retinopathy. Importantly, a drug-mediated rescue of axotomized RGCs of the magnitude seen in this study has not been reported before. This emphasizes the general importance of PKG for neuronal cell death and highlights PKG inhibition as a new therapeutic approach for the treatment of neurodegenerative diseases in general.

\section{cGMP analogues as PKG inhibitors}

Analogues of cGMP carrying an Rp-configurated phosphorothioate modification were first described as exceptionally potent and selective PKG inhibitors in the early 1990s (Butt, Eigenthaler, and Genieser 1994; Butt et al. 1995). While clinically used kinase inhibitors typically block the ATP-binding site present on all kinases (Atkinson et al. 2021; Johnson 2009), cGMP analogues target the cGMP-binding site present only on PKG, i.e., its physiological activation mechanism, thereby affording an extraordinary selectivity for PKG.

With CN238, we identified a cGMP analogue PKG inhibitor that preserved photoreceptor viability and function in $r d 1$ and $r d 10$ retina. This novel compound was found to have improved potency when compared to the reference compound CNO03, a known PKG inhibitor with protective effects in $r d 1, r d 2$, and $r d 10$ mice in vivo (Vighi, Trifunovic, et al. 2018). 
Both cGMP analogues are characterized by the ß-phenyl-1, $\mathrm{N}^{2}$-etheno (PET) group (Wei et al. 1996) and differ only by the additional methyl group in CN238. Interestingly, the compound CNO07 which carried a similar $\mathrm{N}^{2}$-etheno modification was moderately photoreceptor protective, while $\mathrm{CN} 226$, which lacked such a $\mathrm{R}_{2}-\mathrm{R}_{3}$ modification (Figure 1), did not afford photoreceptor protection in $r d 1$ retina. This lack of efficacy was corroborated by the studies on $r d 10$ retina where $\mathrm{CN} 226$ showed significantly less preservation of photoreceptor function than CNO03 or CN238.

The PET-group enhances the lipophilicity of Rp-cGMPS analogues, making it easier for the compounds to reach their target site inside the cell. In addition, the PET-group may bestow the ability to inhibit cyclic nucleotide gated ion (CNG) channels, albeit with an efficacy that is $\approx 2-3 \log$ units lower than for PKG inhibition (Wei et al. 1996). Still, the protective effects of CNO03 and CN238 could potentially stem from both PKG and CNG-channel inhibition. Indeed, CNG channel activity was for many years considered to be a driver of photoreceptor degeneration (Fox, Poblenz, and He 1999; Paquet-Durand et al. 2011). However, numerous studies in the last two decades explored the use of CNG-channel blockers, essentially without tangible results. Moreover, a recent study found that the selective block of CNG-channels with L-cis-diltiazem increased rather than prevented photoreceptor cell death (Das et al. 2020). Together, this makes it seem unlikely that the protective effects of CN003 / CN238 were due to CNG-channel inhibition. Nevertheless, a modulation of also CNG-channel activity by these compounds cannot be entirely excluded at this point.

\section{Effect of PKG inhibitors on photoreceptor function}

The PKG inhibitors CNO03 and CN238 robustly preserved the function of rd10-mutant photoreceptors as assessed via retinal recording of MEA $\mu E R G$ field-potentials. Each of the 59 MEA electrodes covered an area of approx. $80 \mu \mathrm{m}^{2}$, meaning that the field potentials recorded likely originated from thousands of photoreceptors (Stett et al. 2003). Likewise, the amplitude of the initial negative deflection in the $\mu E R G$ represents the sum response of a large number of photoreceptors in a given recording field. Moreover, the recording of $\mu E R G s$ at different retinal locations allowed the creation of spatial activity maps for light responsiveness, both for treated and untreated tissues. The comparison of such maps of $r d 10$ retina revealed large differences between $\mathrm{CNO03/CN238}$ treated and untreated specimens, not only in terms of amplitudes of negative $\mu E R G$ deflections but also in terms of the areas of the retina showing 
responses to light flashes. This in turn demonstrates the magnitude of the photoreceptor protection over a large retinal area, an effect that was corroborated by the histological examination of the retina.

\section{PKG inhibition affords multilevel neuronal protection}

The deleterious effects of high cGMP on photoreceptor viability were established already in the 1970s (Farber and Lolley 1974; Lolley et al. 1977). Yet, the role of PKG as a necessary and sufficient mediator of cGMP-dependent photoreceptor cell death was recognized only more recently (Paquet-Durand et al. 2009; Power et al. 2020). Accordingly, a systematic screening of PKG targeting CGMP analogues in various in vitro and in vivo models identified $\mathrm{CNOO3}$ as a compound that afforded strong functional protection of $r d 1-, r d 2-$, and $r d 10-$ mutant photoreceptors (Vighi, Trifunović, et al. 2018).

The finding that PKG inhibition with either $\mathrm{CNOO3}$ or $\mathrm{CN} 238$, in addition to photoreceptor protection, also prevented the demise of RGCs in both WT and $r d 10$ long-term retinal explant cultures was entirely unexpected. The transection of the optic nerve is a massive insult, known to cause rapid RGC function loss and degeneration (Berkelaar et al. 1994; Osborne et al. 2016). Instead, our MEA recordings indicated a striking preservation of RGC function, concomitant with a marked and significant increase in morphological RGC survival. A recent MEA study on retinal explant cultures found that in WT retina RGC activity gradually decreased to essentially zero within a culture period of 14 days. More importantly, RGC responses to light stimulation were no longer observed beyond 7 days of culture (Alarautalahti et al. 2019), a results that corresponds to our observations on both WT and $r d 10$ retina.

To investigate whether RGCs survival was somehow related to photoreceptor rescue, WT retinal explant cultures were treated with CN238 and compared with untreated specimens. Also, in treated WT retina the MEA recordings revealed strong RGC responses correlated to light stimuli. Immunohistochemical analysis, using the RGC marker RBPMS (Rodriguez, de Sevilla Müller, and Brecha 2014; Wang et al. 2016) and performed on the recorded retinal explants, demonstrated the strongly improved RGC survival after CN238 treatment. Remarkably, the number of RBPMS positive RGCs in both $r d 10$ and WT untreated retinal explants were approximately equal. Furthermore, in WT retina, the comparison of the number of photoreceptors in a row did not show any difference between the CN238-treated and untreated groups. These results suggest that in both the WT and the $r d 10$ situation, long-term 
retinal explant cultures display a loss of RGCs over time and that their rescue by CN238 is independent of photoreceptor survival. The failure of the cGMP analogue CN226 to preserve RGCs viability and function in rd10 retinal explants indicates that RGC survival is connected to PKG inhibition.

The very marked RGC protection seen with CNOO3 and CN238 makes these compounds attractive for therapy development beyond photoreceptor diseases. Indeed, RGC degeneration is a hallmark of several retinal diseases with only limited treatment options to date. This includes glaucoma (Beykin et al. 2021), diabetic retinopathy (Lynch and Abràmoff 2017), exudative age-related macular degeneration (Medeiros and Curcio 2001), and nonexudative age-related macular degeneration (Yenice et al. 2015). How exactly PKG inhibition may afford RGC neuroprotection is not clear at present, yet numerous earlier studies have invoked detrimental effects of nitric oxide synthase and nitric oxide (NO) on RGCs (MuellerBuehl et al. 2021; Neufeld, Sawada, and Becker 1999) also in optic nerve injury (Husain et al. 2014). Since, NO activates soluble guanylyl cyclase to produce cGMP and activate PKG (Bian and Murad 2014) increased NO production in injured RGCs will likely also cause overactivation of PKG, providing a rationale for the use of PKG inhibitors for RGC neuroprotection. The axotomy-induced degeneration of ganglion cells resembles a Wallerian-like retrograde degeneration (Howell et al. 2013; Vrabec and Levin 2007). The fact that PKG inhibition significantly reduces this type of degeneration thus indicates that PKG inhibitors may be applicable even more broadly in neurodegenerative conditions characterized by axonal damage, such as spinal cord injury or multiple sclerosis.

\section{Concluding remarks}

While there has been tremendous progress in the development of new forms of therapy for RD, including gene, molecular and stem cell-based therapies (Hammond et al. 2021; Maguire et al. 2021), as well as retinal prostheses (Kitiratschky et al. 2015), there is still an important unmet medical need for more broadly applicable therapies that may benefit large groups of RD-patients. Our results support the idea that PKG/cGMP signaling is involved in photoreceptor degenerative processes (Power et al. 2020) and identify CN238 as a secondgeneration drug candidate with protective effects on photoreceptor survival and function in two mouse models for RD in vitro. However, further studies need to be conducted to assess whether the protective effects of $\mathrm{CN} 238$ can be extended to other models for RD 
characterized by abnormal cGMP signaling. In addition, the protective effect on RGCs in the explant culture system opens new perspectives for the use of PKG inhibitors for the treatment of common retinal diseases, including glaucoma.

\section{Conflict of interest declaration}

A. R., F. S., and F.P.-D. have filed for three patents on the synthesis and use of cGMP analogues (PCTWO2016/146669A1, PCT/EP2017/066113, and PCT/EP2017/071859) and have obtained a European Medicine Agency orphan drug designation for the use of $\mathrm{CNO}$ for the treatment of retinitis pigmentosa (EU/3/15/1462). F.P.-D. is shareholder of, or has other financial interest in, the company Mireca Medicines, which intends to forward clinical testing of cGMP analogues.

\section{Acknowledgements}

We thank Norman Rieger for excellent technical assistance, Philipp Henning for his help in generating FSS-PKG constructs and Mathias Seeliger, Thomas Euler, Timm, Schubert, Per Ekström, and John Groten for helpful discussions. This research was funded by grants from the European Union (transMed; H2020-MSCA-765441), the Baden-Württemberg Foundation (BWST-WSF_006), the Charlotte and the Tistou Kerstan Foundation, and the German Ministry for Education and Research (BMBF; TargetRD, 16GW0267K, 16GW0269, 16GW0270). 


\section{References}

Alarautalahti, V., S. Ragauskas, J. J. Hakkarainen, H. Uusitalo-Järvinen, H. Uusitalo, J. Hyttinen, G. Kalesnykas, and S. Nymark. 2019. 'Viability of Mouse Retinal Explant Cultures Assessed by Preservation of Functionality and Morphology', Invest Ophthalmol Vis Sci, 60: 1914-27.

Arango-Gonzalez, B., D. Trifunović, A. Sahaboglu, K. Kranz, S. Michalakis, P. Farinelli, S. Koch, F. Koch, S. Cottet, U. Janssen-Bienhold, K. Dedek, M. Biel, E. Zrenner, T. Euler, P. Ekström, M. Ueffing, and F. Paquet-Durand. 2014. 'Identification of a common non-apoptotic cell death mechanism in hereditary retinal degeneration', PLoS One, 9: e112142.

Atkinson, E. L., J. legre, P. D. Brear, E. A. Zhabina, M. Hyvönen, and D. R. Spring. 2021. 'Downfalls of Chemical Probes Acting at the Kinase ATP-Site: CK2 as a Case Study', Molecules, 26.

Belhadj, S., A. Tolone, G. Christensen, S. Das, Y. Chen, and F. Paquet-Durand. 2020. 'Long-Term, Serum-Free Cultivation of Organotypic Mouse Retina Explants with Intact Retinal Pigment Epithelium', J Vis Exp.

Berger, W., B. Kloeckener-Gruissem, and J. Neidhardt. 2010. 'The molecular basis of human retinal and vitreoretinal diseases', Prog Retin Eye Res, 29: 335-75.

Berkelaar, M., D. B. Clarke, Y. C. Wang, G. M. Bray, and A. J. Aguayo. 1994. 'Axotomy results in delayed death and apoptosis of retinal ganglion cells in adult rats', J Neurosci, 14: 4368-74.

Beykin, G., A. M. Norcia, V. J. Srinivasan, A. Dubra, and J. L. Goldberg. 2021. 'Discovery and clinical translation of novel glaucoma biomarkers', Prog Retin Eye Res, 80: 100875.

Bian, K., and F. Murad. 2014. 'What is next in nitric oxide research? From cardiovascular system to cancer biology', Nitric Oxide, 43: 3-7.

Browning, D. D. 2008. 'Protein kinase $\mathrm{G}$ as a therapeutic target for the treatment of metastatic colorectal cancer', Expert Opin Ther Targets, 12: 367-76.

Butt, E., M. Eigenthaler, and H. G. Genieser. 1994. '(Rp)-8-pCPT-cGMPS, a novel cGMP-dependent protein kinase inhibitor', Eur J Pharmacol, 269: 265-8.

Butt, E., D. Pöhler, H. G. Genieser, J. P. Huggins, and B. Bucher. 1995. 'Inhibition of cyclic GMPdependent protein kinase-mediated effects by (Rp)-8-bromo-PET-cyclic GMPS', Br J Pharmacol, 116: 3110-6.

Caffé, A. R., P. Ahuja, B. Holmqvist, S. Azadi, J. Forsell, I. Holmqvist, A. K. Söderpalm, and T. van Veen. 2001. 'Mouse retina explants after long-term culture in serum free medium', Journal of Chemical Neuroanatomy, 22: 263-73.

Canals, S., M. J. Casarejos, S. de Bernardo, E. Rodríguez-Martín, and M. A. Mena. 2003. 'Nitric oxide triggers the toxicity due to glutathione depletion in midbrain cultures through 12lipoxygenase', J Biol Chem, 278: 21542-9.

Canzoniero, L. M., A. Adornetto, A. Secondo, S. Magi, C. Dell'aversano, A. Scorziello, S. Amoroso, and G. Di Renzo. 2006. 'Involvement of the nitric oxide/protein kinase $G$ pathway in polychlorinated biphenyl-induced cell death in SH-SY $5 Y$ neuroblastoma cells', J Neurosci Res, 84: 692-7.

Cook, P. F., M. E. Neville, Jr., K. E. Vrana, F. T. Hartl, and R. Roskoski, Jr. 1982. 'Adenosine cyclic 3',5'monophosphate dependent protein kinase: kinetic mechanism for the bovine skeletal muscle catalytic subunit', Biochemistry, 21: 5794-9.

Das, Soumyaparna, Valerie Popp, Michael Power, Kathrin Groeneveld, Christian Melle, Luke Rogerson, Marlly Achury, Frank Schwede, Torsten Strasser, Thomas Euler, François PaquetDurand, and Vasilica Nache. 2020. 'Redefining the role of $\mathrm{Ca}<$ sup $>2+</$ sup $>$-permeable channels in hereditary photoreceptor degeneration using the $\mathrm{D}$ - and L-cis enantiomers of diltiazem', bioRxiv: 2020.12.04.411827.

Fallahian, F., F. Karami-Tehrani, S. Salami, and M. Aghaei. 2011. 'Cyclic GMP induced apoptosis via protein kinase $\mathrm{G}$ in oestrogen receptor-positive and -negative breast cancer cell lines', Febs $j$, 278: 3360-9.

Farber, D. B., and R. N. Lolley. 1974. 'Cyclic guanosine monophosphate: elevation in degenerating photoreceptor cells of the C3H mouse retina', Science, 186: 449-51. 
bioRxiv preprint doi: https://doi.org/10.1101/2021.08.05.455191; this version posted September 7, 2021. The copyright holder for this preprint (which was not certified by peer review) is the author/funder, who has granted bioRxiv a license to display the preprint in perpetuity. It is made available under aCC-BY-NC-ND 4.0 International license.

Fox, D. A., A. T. Poblenz, and L. He. 1999. 'Calcium overload triggers rod photoreceptor apoptotic cell death in chemical-induced and inherited retinal degenerations', Ann N Y Acad Sci, 893: 282-5.

Hammond, S. M., A. Aartsma-Rus, S. Alves, S. E. Borgos, R. A. M. Buijsen, R. W. J. Collin, G. Covello, M. A. Denti, L. R. Desviat, L. Echevarría, C. Foged, G. Gaina, A. Garanto, A. T. Goyenvalle, M. Guzowska, I. Holodnuka, D. R. Jones, S. Krause, T. Lehto, M. Montolio, W. Van Roon-Mom, and V. Arechavala-Gomeza. 2021. 'Delivery of oligonucleotide-based therapeutics: challenges and opportunities', EMBO Mol Med, 13: e13243.

Han, J., A. Dinculescu, X. Dai, W. Du, W. C. Smith, and J. Pang. 2013. 'Review: the history and role of naturally occurring mouse models with Pde6b mutations', Mol Vis, 19: 2579-89.

Haq, W., J. Dietter, S. Bolz, and E. Zrenner. 2018. 'Feasibility study for a glutamate driven subretinal prosthesis: local subretinal application of glutamate on blind retina evoke network-mediated responses in different types of ganglion cells', J Neural Eng, 15: 045004.

Haq, W., J. Dietter, and E. Zrenner. 2018. 'Electrical activation of degenerated photoreceptors in blind mouse retina elicited network-mediated responses in different types of ganglion cells', Sci Rep, 8: 16998.

Howell, G. R., I. Soto, R. T. Libby, and S. W. John. 2013. 'Intrinsic axonal degeneration pathways are critical for glaucomatous damage', Exp Neurol, 246: 54-61.

Husain, S., Y. Abdul, S. Singh, A. Ahmad, and M. Husain. 2014. 'Regulation of nitric oxide production by $\delta$-opioid receptors during glaucomatous injury', PLoS One, 9: e110397.

Johnson, L. N. 2009. 'Protein kinase inhibitors: contributions from structure to clinical compounds', $Q$ Rev Biophys, 42: 1-40.

Kim, J. J., R. Lorenz, S. T. Arold, A. S. Reger, B. Sankaran, D. E. Casteel, F. W. Herberg, and C. Kim. 2016. 'Crystal Structure of PKG I:cGMP Complex Reveals a cGMP-Mediated Dimeric Interface that Facilitates cGMP-Induced Activation', Structure, 24: 710-20.

Kitiratschky, V. B., K. Stingl, B. Wilhelm, T. Peters, D. Besch, H. Sachs, F. Gekeler, K. U. Bartz-Schmidt, and E. Zrenner. 2015. 'Safety evaluation of "retina implant alpha IMS"--a prospective clinical trial', Graefes Arch Clin Exp Ophthalmol, 253: 381-7.

Leung, E. L., J. C. Wong, M. G. Johlfs, B. K. Tsang, and R. R. Fiscus. 2010. 'Protein kinase G type lalpha activity in human ovarian cancer cells significantly contributes to enhanced Src activation and DNA synthesis/cell proliferation', Mol Cancer Res, 8: 578-91.

Lolley, R. N., D. B. Farber, M. E. Rayborn, and J. G. Hollyfield. 1977. 'Cyclic GMP accumulation causes degeneration of photoreceptor cells: simulation of an inherited disease', Science, 196: 664-6.

Loo, D. T. 2011. 'In situ detection of apoptosis by the TUNEL assay: an overview of techniques', Methods Mol Biol, 682: 3-13.

Lynch, S. K., and M. D. Abràmoff. 2017. 'Diabetic retinopathy is a neurodegenerative disorder', Vision Res, 139: 101-07.

Maguire, A. M., J. Bennett, E. M. Aleman, B. P. Leroy, and T. S. Aleman. 2021. 'Clinical Perspective: Treating RPE65-Associated Retinal Dystrophy', Mol Ther, 29: 442-63.

Medeiros, N. E., and C. A. Curcio. 2001. 'Preservation of ganglion cell layer neurons in age-related macular degeneration', Invest Ophthalmol Vis Sci, 42: 795-803.

Mueller-Buehl, A. M., T. Tsai, J. Hurst, C. Theiss, L. Peters, L. Hofmann, F. Herms, S. Kuehn, S. Schnichels, and S. C. Joachim. 2021. 'Reduced Retinal Degeneration in an Oxidative Stress Organ Culture Model through an iNOS-Inhibitor', Biology (Basel), 10.

Neufeld, A. H., A. Sawada, and B. Becker. 1999. 'Inhibition of nitric-oxide synthase 2 by aminoguanidine provides neuroprotection of retinal ganglion cells in a rat model of chronic glaucoma', Proc Natl Acad Sci U S A, 96: 9944-8.

Osborne, A., M. Hopes, P. Wright, D. C. Broadway, and J. Sanderson. 2016. 'Human organotypic retinal cultures (HORCs) as a chronic experimental model for investigation of retinal ganglion cell degeneration', Exp Eye Res, 143: 28-38.

Paquet-Durand, F., S. Beck, S. Michalakis, T. Goldmann, G. Huber, R. Mühlfriedel, D. Trifunović, M. D. Fischer, E. Fahl, G. Duetsch, E. Becirovic, U. Wolfrum, T. van Veen, M. Biel, N. Tanimoto, and M. W. Seeliger. 2011. 'A key role for cyclic nucleotide gated (CNG) channels in cGMP-related retinitis pigmentosa', Hum Mol Genet, 20: 941-7. 
Paquet-Durand, F., S. M. Hauck, T. van Veen, M. Ueffing, and P. Ekström. 2009. 'PKG activity causes photoreceptor cell death in two retinitis pigmentosa models', J Neurochem, 108: 796-810.

Power, M., S. Das, K. Schütze, V. Marigo, P. Ekström, and F. Paquet-Durand. 2020. 'Cellular mechanisms of hereditary photoreceptor degeneration - Focus on cGMP', Prog Retin Eye Res, 74: 100772.

Rodriguez, A. R., L. P. de Sevilla Müller, and N. C. Brecha. 2014. 'The RNA binding protein RBPMS is a selective marker of ganglion cells in the mammalian retina', J Comp Neurol, 522: 1411-43.

Sahaboglu, A., O. Paquet-Durand, J. Dietter, K. Dengler, S. Bernhard-Kurz, P. A. Ekström, B. Hitzmann, M. Ueffing, and F. Paquet-Durand. 2013. 'Retinitis pigmentosa: rapid neurodegeneration is governed by slow cell death mechanisms', Cell Death Dis, 4: e488.

Sahel, J. A., K. Marazova, and I. Audo. 2014. 'Clinical characteristics and current therapies for inherited retinal degenerations', Cold Spring Harb Perspect Med, 5: a017111.

Sancho-Pelluz, J., B. Arango-Gonzalez, S. Kustermann, F. J. Romero, T. van Veen, E. Zrenner, P. Ekstrom, and F. Paquet-Durand. 2008. 'Photoreceptor cell death mechanisms in inherited retinal degeneration', Mol Neurobiol, 38: 253-69.

Stett, A., U. Egert, E. Guenther, F. Hofmann, T. Meyer, W. Nisch, and H. Haemmerle. 2003. 'Biological application of microelectrode arrays in drug discovery and basic research', Anal Bioanal Chem, 377: 486-95.

Vighi, E., D. Trifunovic, P. Veiga-Crespo, A. Rentsch, D. Hoffmann, A. Sahaboglu, T. Strasser, M. Kulkarni, E. Bertolotti, A. van den Heuvel, T. Peters, A. Reijerkerk, T. Euler, M. Ueffing, F. Schwede, H. G. Genieser, P. Gaillard, V. Marigo, P. Ekstrom, and F. Paquet-Durand. 2018. 'Combination of cGMP analogue and drug delivery system provides functional protection in hereditary retinal degeneration', Proc Natl Acad Sci U S A, 115: E2997-E3006.

Vighi, E., D. Trifunović, P. Veiga-Crespo, A. Rentsch, D. Hoffmann, A. Sahaboglu, T. Strasser, M. Kulkarni, E. Bertolotti, A. van den Heuvel, T. Peters, A. Reijerkerk, T. Euler, M. Ueffing, F. Schwede, H. G. Genieser, P. Gaillard, V. Marigo, P. Ekström, and F. Paquet-Durand. 2018. 'Combination of cGMP analogue and drug delivery system provides functional protection in hereditary retinal degeneration', Proc Natl Acad Sci U S A, 115: E2997-e3006.

Vrabec, J. P., and L. A. Levin. 2007. 'The neurobiology of cell death in glaucoma', Eye (Lond), 21 Suppl 1: S11-4.

Wang, Y., W. Wang, J. Liu, X. Huang, R. Liu, H. Xia, N. C. Brecha, M. Pu, and J. Gao. 2016. 'Protective Effect of ALA in Crushed Optic Nerve Cat Retinal Ganglion Cells Using a New Marker RBPMS', PLoS One, 11: e0160309.

Wei, J. Y., E. D. Cohen, Y. Y. Yan, H. G. Genieser, and C. J. Barnstable. 1996. 'Identification of competitive antagonists of the rod photoreceptor CGMP-gated cation channel: beta-phenyl1,N2-etheno-substituted cGMP analogues as probes of the cGMP-binding site', Biochemistry, 35: 16815-23.

Yenice, E., A. Şengün, G. Soyugelen Demirok, and E. Turaçlı. 2015. 'Ganglion cell complex thickness in nonexudative age-related macular degeneration', Eye (Lond), 29: 1076-80.

Zhao, J., J. Trewhella, J. Corbin, S. Francis, R. Mitchell, R. Brushia, and D. Walsh. 1997. 'Progressive cyclic nucleotide-induced conformational changes in the cGMP-dependent protein kinase studied by small angle X-ray scattering in solution', J Biol Chem, 272: 31929-36. 


\section{Supplementary information}

\section{PKG1 $\alpha$}

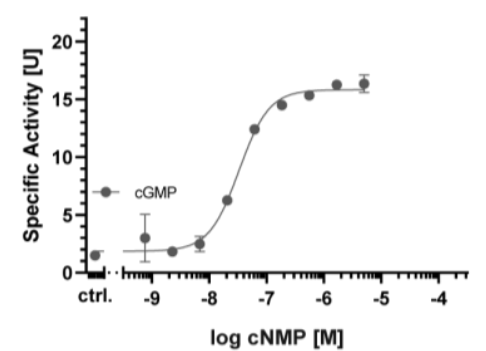

PKG1 $\beta$

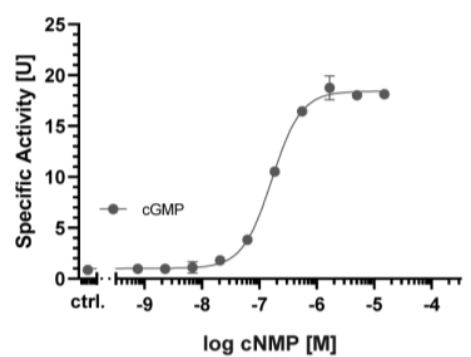

PKG2

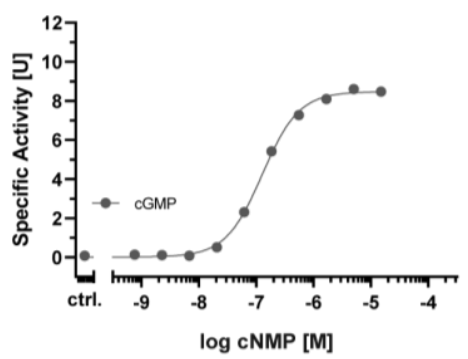

$\mathrm{K}_{\mathrm{act}}=28.6 \pm 3 \mathrm{nM}(6)$

$\mathrm{K}_{\mathrm{act}}=141 \pm 12 \mathrm{nM}(6)$

Figure S1: cGMP-dependent activation of the three distinct PKG isoforms. Activation curves of PKG1 $\alpha, P K G 1 \beta$, and PKG2 were obtained with cGMP dilution series ranging from $5 \mu \mathrm{M}$ to 25.4 pm for PKG1 $\alpha$ and from $15 \mu \mathrm{M}$ to 762 pm for PKG1 $\beta$ and PKG2. 


\begin{tabular}{|c|c|c|c|}
\hline & PKG1a & PKG1及 & PKG2 \\
\hline ஜ̊ & 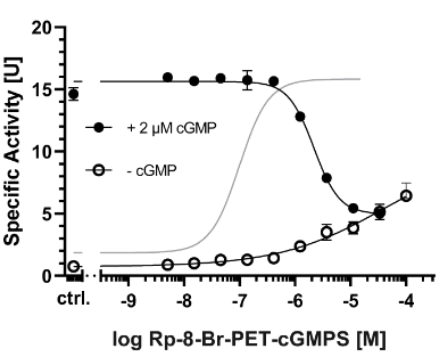 & 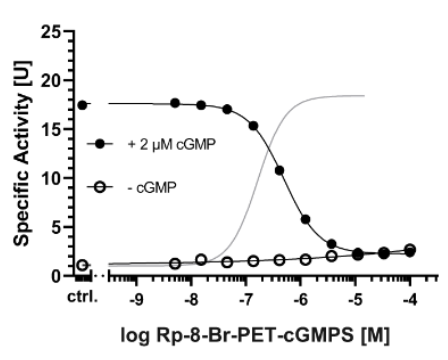 & 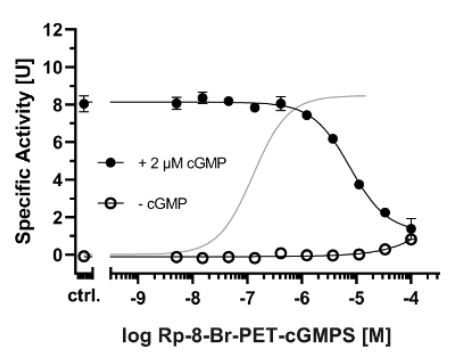 \\
\hline $\mathrm{IC}_{50}$ & $1.9 \pm 0.24 \mu \mathrm{M}(3)$ & $0.40 \pm 0.09 \mu \mathrm{M}(3)$ & $5.8 \pm 1.7 \mu \mathrm{M}(3)$ \\
\hline$\underset{\cup}{\stackrel{\infty}{Z}}$ & (log Rp-8-Br-pMe-PET-CGMPS [M] & log Rp-8-Br-pMe-PET-CGMPS [M] & $\begin{array}{lll} & \ldots \\
\log \text { Rp-8-Br-pMe-PET-CGMPS [M] }\end{array}$ \\
\hline $\mathrm{IC}_{50}$ & $1.1 \pm 0.2 \mu \mathrm{M}(5)$ & $0.38 \pm 0.22 \mu \mathrm{M}(3)$ & $4.6 \pm 1.0 \mu \mathrm{M}(3)$ \\
\hline$\underset{\text { N }}{\stackrel{N}{Z}}$ & (log Rp-8-PAmdMT-CGMPS [M] & 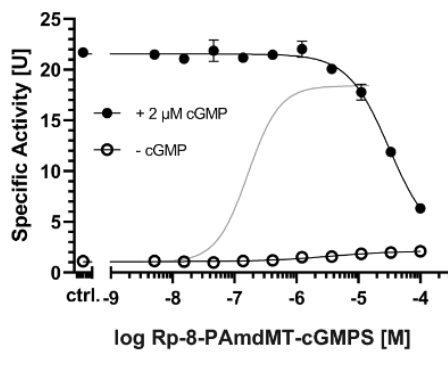 & 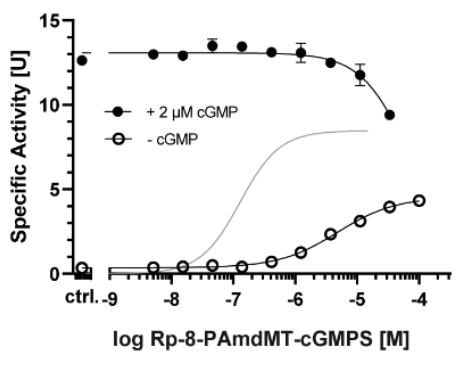 \\
\hline $\mathrm{IC}_{50}$ & $-(2)\left(K_{\text {act }}=0.87 \pm 0.1 \mu \mathrm{M}\right)$ & (2) & $-(2)$ \\
\hline
\end{tabular}

Figure S2: Agonistic and antagonistic properties of the cGMP analogues CN003, CN238, and CN226. The activation of all PKG isoforms (grey solid line) was determined with cGMP (cf. supplementary figure 1). Activation/Inhibition curves of $5 \mathrm{nM}$ PKG1 $\alpha$, PKG1 $\beta$, and PKG2 were measured with the CGMP analogues CN003, CN238, and CN226. For each analogue first the activation was determined (open circles) and in a second approach the inhibition in the presence of $2 \mu \mathrm{M}$ cGMP was measured (solid circles). IC 50 values were calculated from cGMP analogue dilution series ranging from $100 \mu \mathrm{M}$ to $5.1 \mathrm{nM}$ obtained in 2-5 independent measurements. 